\title{
Computational exploration of two-dimensional silicon diarsenide and germanium arsenide for photovoltaic applications
}

\author{
Sri Kasi Matta, Chunmei Zhang, Yalong Jiao, Anthony O'Mullane and Aijun Du*
}

\author{
Full Research Paper \\ Address: \\ School of Chemistry, Physics and Mechanical Engineering, \\ Queensland University of Technology, Garden Point Campus, QLD \\ 4001, Brisbane, Australia \\ Email: \\ Aijun Du* - aijun.du@qut.edu.au \\ * Corresponding author \\ Keywords: \\ density functional theory (DFT); photovoltaic applications; solar cell; \\ two-dimensional semiconductors \\ Beilstein J. Nanotechnol. 2018, 9, 1247-1253. \\ doi:10.3762/bjnano.9.116 \\ Received: 27 November 2017 \\ Accepted: 29 March 2018 \\ Published: 19 April 2018 \\ Associate Editor: N. Motta \\ (c) 2018 Matta et al.; licensee Beilstein-Institut. \\ License and terms: see end of document.
}

\section{Open Access}

\begin{abstract}
The properties of bulk compounds required to be suitable for photovoltaic applications, such as excellent visible light absorption, favorable exciton formation, and charge separation are equally essential for two-dimensional (2D) materials. Here, we systematically study 2D group IV-V compounds such as $\mathrm{SiAs}_{2}$ and $\mathrm{GeAs}_{2}$ with regard to their structural, electronic and optical properties using density functional theory (DFT), hybrid functional and Bethe-Salpeter equation (BSE) approaches. We find that the exfoliation of single-layer $\mathrm{SiAs}_{2}$ and $\mathrm{GeAs}_{2}$ is highly feasible and in principle could be carried out experimentally by mechanical cleavage due to the dynamic stability of the compounds, which is inferred by analyzing their vibrational normal mode. $\mathrm{SiAs}_{2}$ and GeAs$_{2}$ monolayers possess a bandgap of 1.91 and $1.64 \mathrm{eV}$, respectively, which is excellent for sunlight harvesting, while the exciton binding energy is found to be 0.25 and $0.14 \mathrm{eV}$, respectively. Furthermore, band-gap tuning is also possible by application of tensile strain. Our results highlight a new family of 2D materials with great potential for solar cell applications.
\end{abstract}

\section{Introduction}

The potential applications of two-dimensional (2D) materials are one of the key research areas for many researchers since graphene was isolated and characterized in 2004 [1]. The number of applications is vast including photovoltaics, nanoelectronics, Dirac materials and solar fuels through water splitting, to name but a few. Although single elemental 2D materials from groups IV and V, such as like phosphorene and stanine, have been studied in detail [2,3], we focus our study on the less well-known combination of IV-V compound semiconductor materials at the two-dimensional scale for their possible electronic applications. The less extensively studied compounds $\mathrm{SiAs}_{2}$ and $\mathrm{GeAs}_{2}$ are considered here for a computational study. The synthesis of these compounds [4,5] was reported a few decades ago as well as their basic crystal structures and corresponding phase diagrams $[6,7]$.

In 1963, Hulliger et al. [8] predicted that the group IV-V compounds $\mathrm{SiAs}$, GeAs and $\mathrm{GeAs}_{2}$ would show semiconductor be- 
havior. The synthesis and crystal structures of the group IV- $-\mathrm{V}_{2}$ type compounds $\mathrm{SiP}_{2}, \mathrm{SiAs}_{2}$ and $\mathrm{GeAs}_{2}$ were reported later. All three compounds exhibit the orthorhombic space group Pbam with eight formula units per cell [4]. However, they did not report the band structure or the band gap values of these materials. Later, Wu et al. performed theoretical studies on silicon and germanium arsenides [9] to predict and reaffirm that $\mathrm{m}-\mathrm{SiAs} /$ GeAs and $\mathrm{o}-\mathrm{SiAs}_{2} / \mathrm{GeAs}_{2}$ are indeed semiconductors. The studies were based on band-structure calculations and are in agreement with experimental observations.

A recently reported computational study on $2 \mathrm{D} \mathrm{GeAs}_{2}$ was performed to investigate its thermal conductivity and its suitability for thermoelectric applications [10]. In order to further study $\mathrm{GeAs}_{2}$ and to compare it with a similar material from the same IV-V group combination, we focus our study on two-dimensional $\mathrm{SiAs}_{2}$ and $\mathrm{GeAs}_{2}$ and compare them with their bulk counter parts with regard to electronic band structure, phononvibration frequencies, optical properties, band gap modulation behavior and predict their potential applications.

\section{Computational Details}

Density functional theory (DFT) using plane wave Vienna ab initio simulation package (VASP) code is used for first-principle calculations for this study $[11,12]$. The geometry optimisation is done with generalized gradient approximation in the Perdew-Burke-Ernzerhof form (GGA-PBE) exchange-correlation functional [13]. A Monkhorst-Pack $k$-points grid [14] of $9 \times 3 \times 2$ and $9 \times 3 \times 1$ was used for sampling the first Brillouin zone for bulk and monolayer for geometry optimizations. Both bulk and monolayer systems are set for relaxing until residual force and energy converged to $0.005 \mathrm{eV} / \AA$ and $10^{-7} \mathrm{eV}$, respectively. To study $2 \mathrm{D}$ monolayer systems under periodic boundary conditions, a vacuum layer of about $15 \AA$ was introduced to minimize the spurious interaction between neighboring layers. The electronic band structure is predicted through hybrid density functional theory based on the Heyd-Scuseria-Ernzerhof (HSE) exchange-correlation functional $[15,16]$ and Wannier90 package [17] implemented in the VASP code. The thermodynamic stability of the material is assessed by the formation energy for the $2 \mathrm{D}$ material and is indicated as "the difference in free energy of the 2D material and the lowest value of the bulk equivalent of the same material", $E_{\mathrm{f}}$ :

$$
E_{\mathrm{f}}=\frac{E_{2 \mathrm{D}}}{n_{2 \mathrm{D}}}-\frac{E_{3 \mathrm{D}}}{n_{3 \mathrm{D}}},
$$

where, $E_{2 \mathrm{D}}$ and $E_{3 \mathrm{D}}$ are the energies of the monolayer and the bulk material, respectively. $n_{2 \mathrm{D}}$ and $n_{3 \mathrm{D}}$ are the number of atoms present in the unit cells considered for the calculations
[18-20]. The optical properties are evaluated by determining the frequency-dependent dielectric tensor matrix through hybrid DFT based on the Heyd-Scuseria-Ernzerhof (HSE) exchange-correlation functional $[15,16]$, implemented in VASP [21-23]. The corrected average electrostatic potential in the unit cell with regard to the vacuum potential is obtained from HSE band calculations. It was subsequently used as a generic reference for aligning the band positions that were obtained from HSE06-Wannier calculations. A zero-damped van der Waals correction was incorporated using the DFT-D3 method of Grimme's scheme [24,25] to better describe non-covalent bonding interactions. The projector-augmented-wave (PAW) method [26] was used to describe the electron-ion interaction. Also, the plane-wave energy cut-off was set to ca. $255 \mathrm{eV}$ and, in addition, a high precision option ( $\mathrm{PREC}=$ high) is used in the input file, which would further set cut-off energy and other defaults such as grid spacing representing the augmentation charges, charge densities and potentials (NGFX, NGFY, NGFZ) so as to get accurate results. The phonon spectrum was computed using the density functional perturbation theory (DFPT) [27] as implemented in the Quantum-ESPRESSO package [28]. The excitonic properties are studied using Green's function, GW-Bethe-Salpeter equation (BSE) approach implemented in VASP code [23,29-31]. The GW calculations were performed with a $13 \times 5 \times 1 \mathrm{k}$-grid, the energy cut-off for response function is set at 100 both in GW and BSE approach for exact compatibility. Over and above the GW results, the BSE method was adopted to obtain the light absorption spectrum [21,22] and the optical band gap. BSE was solved by using the ten highest valance bands and ten lowest conduction bands and with a $13 \times 5 \times 1 k$-grid. Using the band gaps obtained from GW and BSE functional methods the exciton binding energy was obtained [32].

\section{Results and Discussion}

The crystal structures of $\mathrm{SiAs}_{2}$ and $\mathrm{GeAs}_{2}$ are orthorhombic with the space group Pbam (no. 55) having eight symmetry operators or atoms in the unit cell [4]. Figure 1 shows the side views of the bulk configurations of $\mathrm{SiAs}_{2}$ and $\mathrm{GeAs}_{2}$ with atomic layers interacting with neighboring layers through weak van der Waals forces. Detailed structural parameters for bulk and monolayers of $\mathrm{SiAs}_{2}$ and $\mathrm{GeAs}_{2}$ are listed in Table 1. The calculated lattice constants for the bulk materials are in good agreement with previous experimental values [4]. The calculated lattice constants for single-layered $\mathrm{SiAs}_{2}$ and $\mathrm{GeAs}_{2}$ are slightly smaller than those calculated for the bulk phases. The lattice constant $c$ is kept constant and greater than $15 \AA$ by adding a vacuum layer.

The dynamical stability of $\mathrm{SiAs}_{2}$ and $\mathrm{GeAs}_{2}$ monolayers is evaluated by analysing the phonon band spectrum. As shown in 


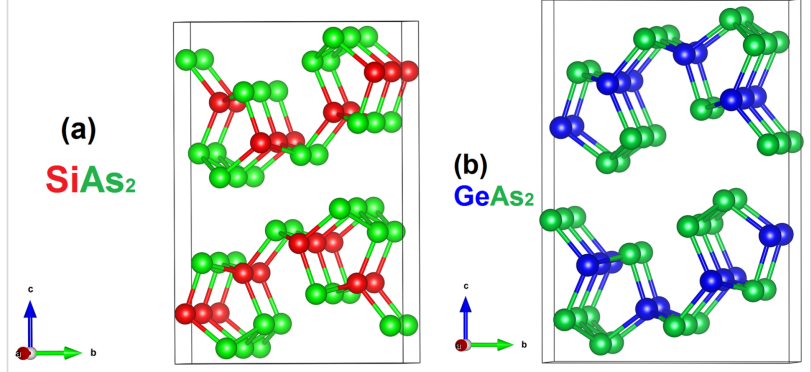

Figure 1: Crystal structure side view of (a) $\mathrm{SiAs}_{2}$ bulk ( $3 \times 2$ super cells) and (b) $\mathrm{GeAs}_{2}$ bulk (red: silicon, blue: germanium, green: arsenic).

Figure 2a and Figure 2b, no imaginary frequency can be found at any wave vector, confirming that the single-layer $\mathrm{SiAs}_{2}$ and $\mathrm{GeAs}_{2}$ are dynamically stable. The thermodynamic stability identified through the energy of formation, $E_{\mathrm{f}}$, calculated from Equation 1 determines the strength of van der Waals interactions in the bulk materials of $\mathrm{SiAs}_{2}$ and $\mathrm{GeAs}_{2}$. Thus, the lower the formation energy the easier it can be extracted from the bulk material. It has been reported that less than $200 \mathrm{meV}$ /atom is considered to be a sufficiently low formation energy that the free-standing 2D layer can be extracted from the bulk material [20]. In our calculations, $E_{\mathrm{f}}$ for $\mathrm{SiAs}_{2}$ and $\mathrm{GeAs}_{2}$ is found to be 66.8 and $76.0 \mathrm{meV} /$ atom, respectively. These values are smaller than the $E_{\mathrm{f}}$ values of already exfoliated $2 \mathrm{D}$ materials. The values are compared with the $E_{f}$ values reported for chalcogenides of $\mathrm{Sn}$ and $\mathrm{Pb}[20,35]$ and are depicted in Figure 2c. According to this evaluation, the exfoliation of monolayer for these materials from their bulk forms is highly feasible.

The band structures determined by HSE-Wannier calculations demonstrate that bulk, bilayer and monolayer structures of $\mathrm{SiAs}_{2}$ and $\mathrm{GeAs}_{2}$ (Figure 3a-d) are semiconductors with indirect band gaps (the VBM and CBM locations are marked). The bandgaps are given in Table 2. These results are consistent with previously reported calculated values for both bulk and monolayers of $\mathrm{GeAs}_{2}$ with values of 0.99 and $1.64 \mathrm{eV}$, respectively [10]. The decrease in the thickness of both $\mathrm{SiAs}_{2}$ and $\mathrm{GeAs}_{2}$ leads to a quantum confinement effect [36] and thus the band gap is increased significantly from the bulk to the monolayer structures in both materials. In addition, the experimentally reported indirect band gap of $1.06 \mathrm{eV}$ by Rau et al. for single crystal orthorhombic $\mathrm{GeAs}_{2}$ is in agreement with our calculated result for the bulk material [5].

The ability of a GeAs $s_{2}$ monolayer to harvest solar light in the visible region is higher, both in absorption intensity and in the range of wavelengths covered, than that of $\mathrm{SiAs}_{2}$. This is shown in Figure 4, which shows the light absorption spectrum calculated from HSE functional in the visible light region (approx. 350-800 nm) compared with the AM1.5G solar spectrum. However, both materials exhibit an almost equal absorption in the

Table 1: Calculated structural parameters of $\mathrm{SiAs}_{2}$ and $\mathrm{GeAs}_{2}$ compared with the experimental values.

\begin{tabular}{|c|c|c|c|c|c|c|}
\hline & \multicolumn{3}{|c|}{$\mathrm{SiAs}_{2}$} & \multicolumn{3}{|c|}{$\mathrm{GeAs}_{2}$} \\
\hline & bulk (calc.) & bulk (exp.) & monolayer (calc.) & bulk (calc.) & bulk (exp.) & monolayer (calc.) \\
\hline$a(\AA)$ & 3.691 & $3.636[4]$ & 3.676 & 3.795 & $\begin{array}{c}3.728[33] \\
(3.721[34])\end{array}$ & 3.760 \\
\hline$b(\AA)$ & 10.124 & $10.37[4]$ & 10.258 & 10.362 & $\begin{array}{c}10.16[33] \\
(10.12[34])\end{array}$ & 10.397 \\
\hline$c(\AA)$ & 14.857 & $14.53[4]$ & - & 14.666 & $\begin{array}{c}14.76[33] \\
(14.74[34])\end{array}$ & - \\
\hline
\end{tabular}
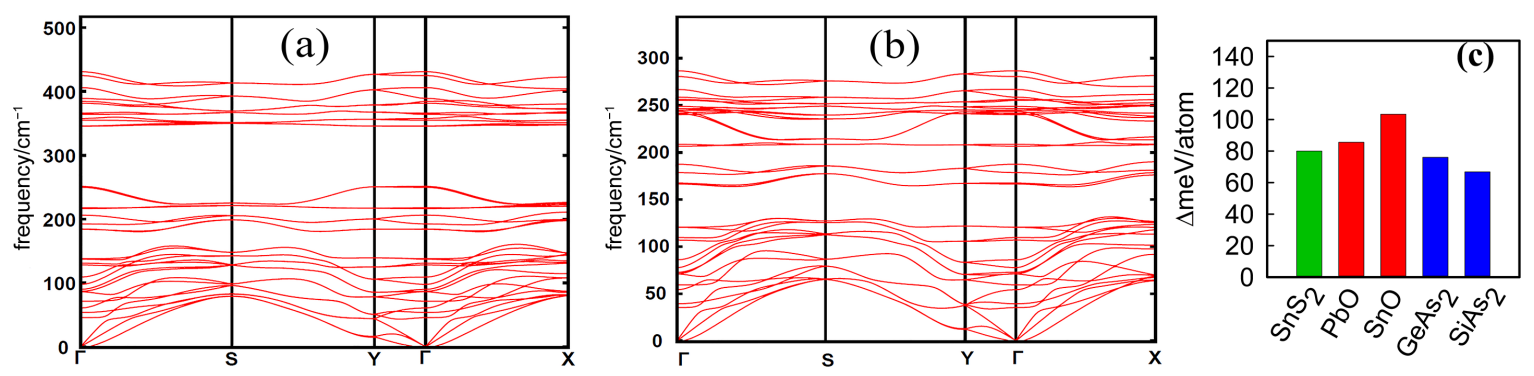

Figure 2: Phonon band structure of a monolayer of (a) $\mathrm{SiAs}_{2}$ and (b) GeAs$s_{2}$ along the high-symmetry points in the 1st Brillouin zone. (c) Energy of formation of the $2 \mathrm{D}$ material from its bulk counterparts (green: experimentally synthesized $2 \mathrm{D}$ compound, red: not experimentally synthesized $2 \mathrm{D}$ compound, blue: possibility to synthesize 2D compound theoretically shown). 

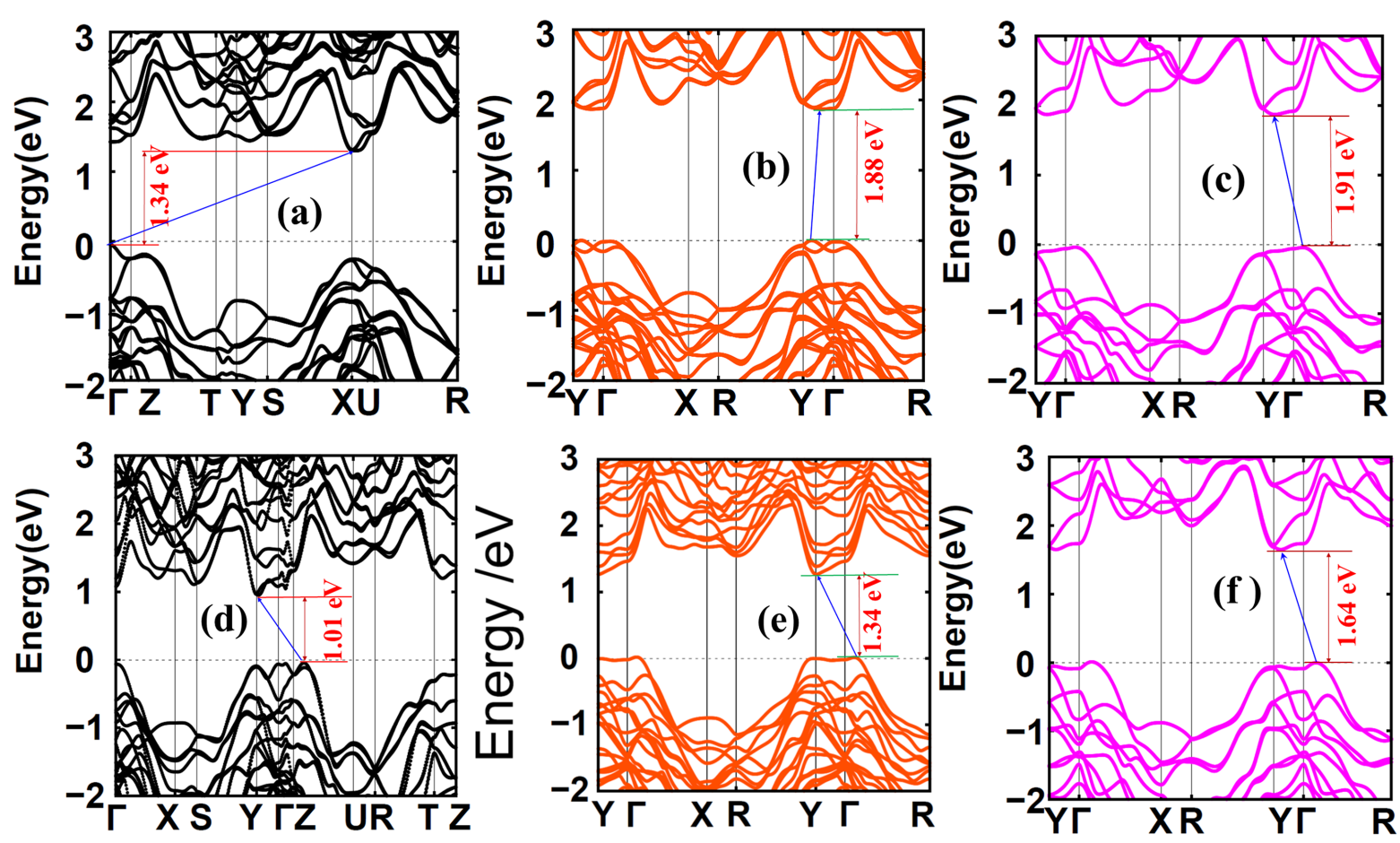

Figure 3: Band structure for $\mathrm{SiAs}_{2}$ and $\mathrm{GeAs}_{2}$ calculated by the HSE-Wannier function method. The Fermi level is set as zero. (a-c) Bulk, bilayer and monolayer of $\mathrm{SiAs}_{2}$, respectively; $(\mathrm{d}-\mathrm{f})$ bulk, bilayer and monolayer of $\mathrm{GeAs}_{2}$, respectively.

Table 2: Calculated band gaps of $\mathrm{SiAs}_{2}$ and $\mathrm{GeAs}_{2}$ for bulk, bilayers and monolayers.

\begin{tabular}{|c|c|c|c|c|c|c|}
\hline & \multicolumn{3}{|c|}{$\mathrm{SiAs}_{2}$} & \multicolumn{3}{|c|}{$\mathrm{GeAs}_{2}$} \\
\hline & bulk & bilayer & monolayer & bulk & bilayer & monolayer \\
\hline bandgap (eV) & 1.34 & 1.86 & 1.91 & 0.99 & 1.34 & 1.64 \\
\hline
\end{tabular}

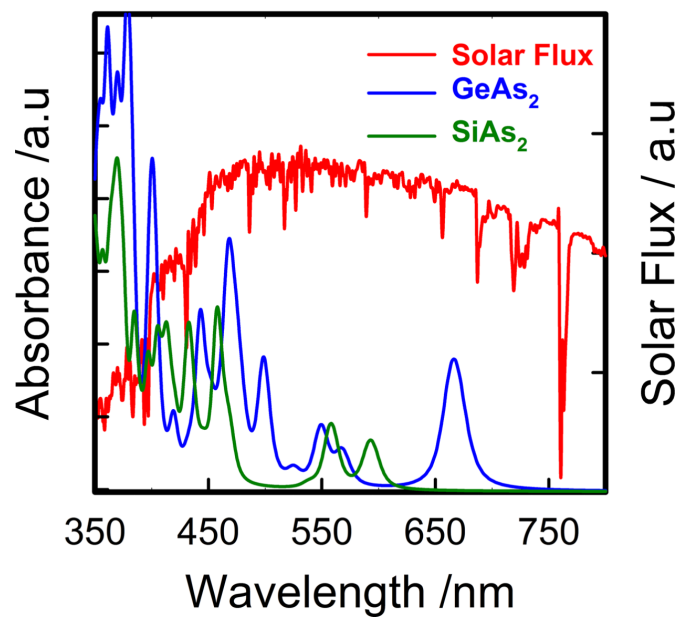

Figure 4: Calculated light absorption spectrum of monolayers of $\mathrm{SiAs}_{2}$ (green) and $\mathrm{GeAs}_{2}$ (blue) using HSE functional superimposed to the incident $\mathrm{AM} 1.5 \mathrm{G}$ solar flux. wavelength region of 350-600 nm. Above this, GeAs 2 still has good absorption up to around $700 \mathrm{~nm}$.

It has been reported that exterior strain on semiconductor nanostructures, especially at the two-dimensional level, influences the electronic properties and the corresponding optical properties $[37,38]$. We, therefore, studied the PBE functional band gap variation as a function of tensile strain (Figure S1, Supporting Information File 1). The band gap variation depends on the viewing direction along the lattice. In the laboratory, an external strain can be imparted by different means such as adlayer-substrate lattice mismatch, external loading, bending or by applying stress on the material [39-42]. While the expansion or compressive strain in the direction of $a$ can transform the semiconductor into a metal [18]. Higher strains (deduced by extrapolation, not shown in the figure) and compression can cause a continuous increase in the band gap from compressive strain to expansion strain in both $\mathrm{SiAs}_{2}$ and $\mathrm{GeAs}_{2}$. This indicates that there is the possibility of band gap tuning to make the semiconductor 
suitable for a desired electronic application. However, this band gap variation was calculated using a PBE functional that is known to underestimate the value of the band gap. However, for the purpose of the illustrating the dependence of the band gap variation on strain, these calculations can be helpful.

The quasi-particle band gaps of $\mathrm{SiAs}_{2}$ and $\mathrm{GeAs}_{2}$ monolayer compounds were found to be 2.26 and $1.86 \mathrm{eV}$, respectively (Figure 5). These values are comparable with the band gaps obtained by using the HSE-Wannier method (1.91 and $1.64 \mathrm{eV}$ for $\mathrm{SiAs}_{2}$ and $\mathrm{GeAs}_{2}$, respectively) with similar and negligible variation amongst the two methods. The corresponding first absorption peaks in the absorption spectra are the optical band gaps at 2.01 and $1.72 \mathrm{eV}$, respectively. Thus the calculated exciton binding energies [43-45] are 0.25 and $0.14 \mathrm{eV}$ for $\mathrm{SiAs}_{2}$ and $\mathrm{GeAs}_{2}$, respectively. Semiconductors with exciton energies in this range of a few hundred millielectronvolts are supposed to play a key role in photovoltaic applications [46].

\section{Conclusion}

We have presented 2D monolayer compounds of $\mathrm{SiAs}_{2}$ and $\mathrm{GeAs}_{2}$ as promising light harvesting semiconductor materials for solar cell applications. The extraction of a monolayer of these materials is likely to be feasible by mechanical exfoliation. Moreover, the calculated phonon spectrum reveals its high dynamical stability for both materials. Additionally, the exciton binding energies are quite low and are comparable to quantum dot semiconductors. It might be possible that these semiconductors could be synthesized as quantum dots and studied in further detail. Band gap tuning appears also possible and could be used to tailor the compounds for various electronic applications.

\section{Supporting Information}

Supporting Information shows the band gap variation as a function of tensile strain.

\section{Supporting Information File 1}

Additional computational data.

[https://www.beilstein-journals.org/bjnano/content/ supplementary/2190-4286-9-116-S1.pdf]

\section{Acknowledgements}

We acknowledge generous grants of high-performance computer time from computing facility at the Queensland University of Technology, The Pawsey Supercomputing Centre and Australian National Facility. A.D. greatly appreciates the financial support of Australian Research Council under Discovery Project (DP130102420 and DP170103598).
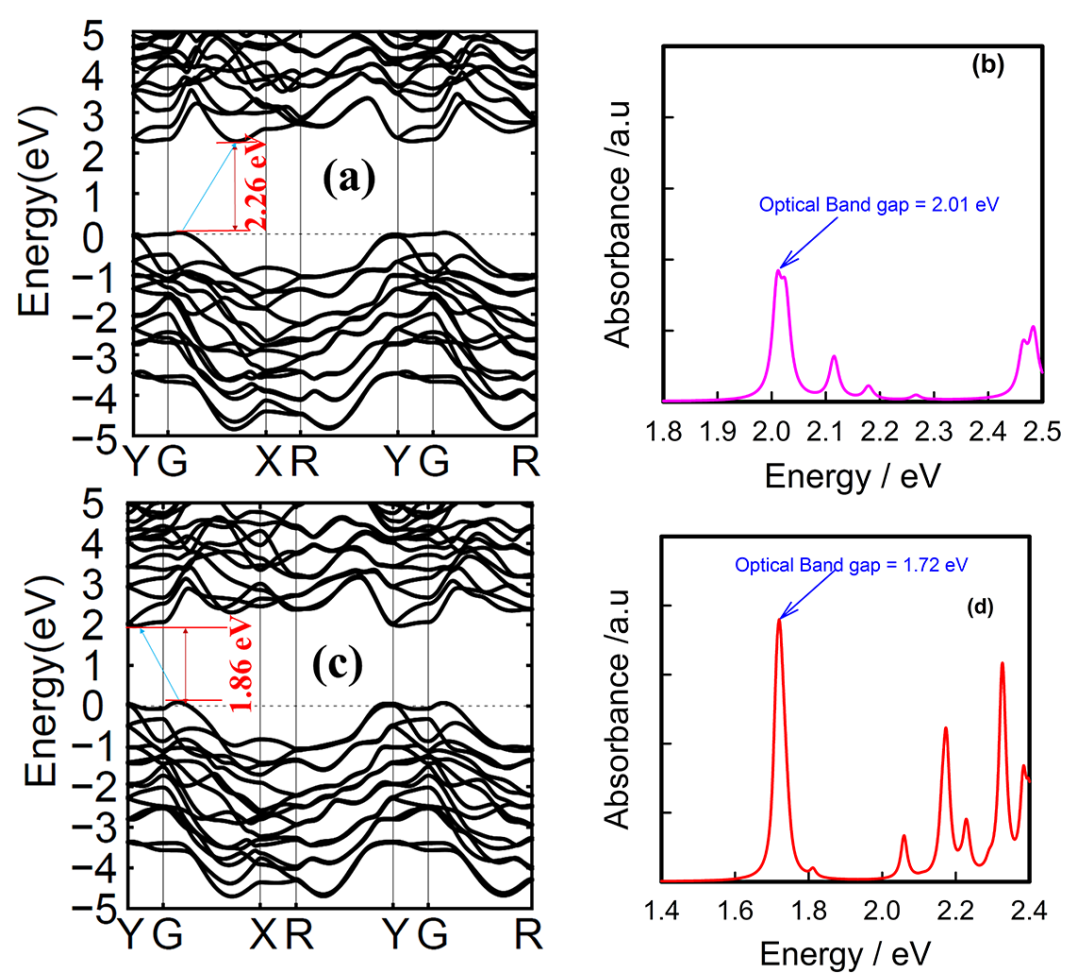

Figure 5: (a,c) GW-band structures and (b,d) BSE-optical absorption spectra of $\mathrm{SiAs}_{2}$ and $\mathrm{GeAs}_{2}$, respectively. 


\section{$\mathrm{ORCID}^{\circledR}$ iDs}

Sri Kasi Matta - https://orcid.org/0000-0003-4465-640X

\section{References}

1. Novoselov, K. S.; Geim, A. K.; Morozov, S. V.; Jiang, D.; Zhang, Y.; Dubonos, S. V.; Grigorieva, I. V.; Firsov, A. A. Science 2004, 306, 666-669. doi:10.1126/science.1102896

2. Garcia, J. C.; de Lima, D. B.; Assali, L. V. C.; Justo, J. F. J. Phys. Chem. C 2011, 115, 13242-13246. doi:10.1021/jp203657w

3. Carvalho, A.; Wang, M.; Zhu, X.; Rodin, A. S.; Su, H.; Castro Neto, A. H. Nat. Rev. Mater. 2016, 1, 16061. doi:10.1038/natrevmats.2016.61

4. Wadsten, T. Acta Chem. Scand. 1967, 21, 593-594. doi:10.3891/acta.chem.scand.21-0593

5. Rau, J. W.; Kannewurf, C. R. Phys. Rev. B 1971, 3, 2581-2587. doi:10.1103/PhysRevB.3.2581

6. Panish, M. B. J. Electrochem. Soc. 1966, 113, 1226-1228. doi:10.1149/1.2423790

7. Ugai, Y. A.; Popov, A. E.; Goncharov, E. G.; Tolubaev, K. G. Zh. Neorg. Khim. 1983, 28, 2944-2947.

8. Hulliger, F.; Mooser, E. J. Phys. Chem. Solids 1963, 24, 283-295. doi:10.1016/0022-3697(63)90133-1

9. Wu, P.; Huang, M. Phys. Status Solidi B 2016, 253, 862-867. doi:10.1002/pssb.201552598

10. Zhao, T.; Sun, Y.; Shuai, Z.; Wang, D. Chem. Mater. 2017, 29, 6261-6268. doi:10.1021/acs.chemmater.7b01343

11. Kresse, G.; Furthmüller, J. Comput. Mater. Sci. 1996, 6, 15-50. doi:10.1016/0927-0256(96)00008-0

12. Kresse, G.; Furthmüller, J. Phys. Rev. B 1996, 54, 11169-11186. doi:10.1103/PhysRevB.54.11169

13. Perdew, J. P.; Burke, K.; Ernzerhof, M. Phys. Rev. Lett. 1996, 77, 3865-3868. doi:10.1103/PhysRevLett.77.3865

14. Monkhorst, H. J.; Pack, J. D. Phys. Rev. B 1976, 13, 5188-5192. doi:10.1103/PhysRevB.13.5188

15. Heyd, J.; Scuseria, G. E.; Ernzerhof, M. J. Chem. Phys. 2003, 118, 8207-8215. doi:10.1063/1.1564060

16. Krukau, A. V.; Vydrov, O. A.; Izmaylov, A. F.; Scuseria, G. E. J. Chem. Phys. 2006, 125, 224106. doi:10.1063/1.2404663

17. Mostofi, A. A.; Yates, J. R.; Pizzi, G.; Lee, Y.-S.; Souza, I.; Vanderbilt, D.; Marzari, N. Comput. Phys. Commun. 2014, 185, 2309-2310. doi:10.1016/j.cpc.2014.05.003

18. Jiao, Y.; Zhou, L.; Ma, F.; Gao, G.; Kou, L.; Bell, J.; Sanvito, S.; Du, A. ACS Appl. Mater. Interfaces 2016, 8, 5385-5392. doi:10.1021/acsami.5b12606

19. Zhuang, H. L.; Hennig, R. G. Chem. Mater. 2013, 25, 3232-3238. doi:10.1021/cm401661x

20. Singh, A. K.; Mathew, K.; Zhuang, H. L.; Hennig, R. G. J. Phys. Chem. Lett. 2015, 6, 1087-1098. doi:10.1021/jz502646d

21. Bernardi, M.; Palummo, M.; Grossman, J. C. Nano Lett. 2013, 13, 3664-3670. doi:10.1021/nl401544y

22. Yang, L.; Deslippe, J.; Park, C.-H.; Cohen, M. L.; Louie, S. G. Phys. Rev. Lett. 2009, 103, 186802. doi:10.1103/PhysRevLett.103.186802

23. Albrecht, S.; Reining, L.; Del Sole, R.; Onida, G. Phys. Rev. Lett. 1998, 80, 4510-4513. doi:10.1103/PhysRevLett.80.4510

24. Grimme, S. J. Comput. Chem. 2006, 27, 1787-1799. doi:10.1002/jcc.20495

25. Grimme, S.; Antony, J.; Ehrlich, S.; Krieg, H. J. Chem. Phys. 2010, 132, 154104. doi:10.1063/1.3382344
26. Blöchl, P. E. Phys. Rev. B 1994, 50, 17953-17979. doi:10.1103/PhysRevB.50.17953

27. Baroni, S.; De Gironcoli, S.; Dal Corso, A.; Giannozzi, P. Rev. Mod. Phys. 2001, 73, 515-562. doi:10.1103/RevModPhys.73.515

28. Giannozzi, P.; Baroni, S.; Bonini, N.; Calandra, M.; Car, R.; Cavazzoni, C.; Ceresoli, D.; Chiarotti, G. L.; Cococcioni, M.; Dabo, I.; Dal Corso, A.; de Gironcoli, S.; Fabris, S.; Fratesi, G.; Gebauer, R.; Gerstmann, U.; Gougoussis, C.; Kokalj, A.; Lazzeri, M.; Martin-Samos, L.; Marzari, N.; Mauri, F.; Mazzarello, R.; Paolini, S.; Pasquarello, A.; Paulatto, L.; Sbraccia, C.; Scandolo, S.; Sclauzero, G.; Seitsonen, A. P.; Smogunov, A.; Umari, P.; Wentzcovitch, R. M. J. Phys.: Condens. Matter 2009, 21, 395502. doi:10.1088/0953-8984/21/39/395502

29. Benedict, L. X.; Shirley, E. L.; Bohn, R. B. Phys. Rev. Lett. 1998, 80 , 4514-4517. doi:10.1103/PhysRevLett.80.4514

30. Onida, G.; Reining, L.; Rubio, A. Rev. Mod. Phys. 2002, 74, 601-659. doi:10.1103/RevModPhys.74.601

31. Rohlfing, M.; Louie, S. G. Phys. Rev. Lett. 1998, 81, 2312-2315. doi:10.1103/PhysRevLett.81.2312

32. Rohlfing, M.; Louie, S. G. Phys. Rev. B: Condens. Matter Mater. Phys. 2000, 62, 4927-4944. doi:10.1103/PhysRevB.62.4927

33. Bryden, J. H. Acta Crystallogr. 1962, 15, 167-171. doi:10.1107/S0365110X62000407

34. Hulliger, F. Structural Chemistry of Layer-Type Phases; Springer: Berlin, Germany, 1976. doi:10.1007/978-94-010-1146-4

35. Singh, A. K.; Hennig, R. G. Appl. Phys. Lett. 2014, 105, 042103. doi:10.1063/1.4891230

36. Cai, Y.; Zhang, G.; Zhang, Y.-W. Sci. Rep. 2014, 4, 6677. doi:10.1038/srep06677

37. Zhou, M.; Duan, W.; Chen, Y.; Du, A. Nanoscale 2015, 7, 15168-15174. doi:10.1039/C5NR04431F

38. Ma, F.; Zhou, M.; Jiao, Y.; Gao, G.; Gu, Y.; Bilic, A.; Chen, Z.; Du, A. Sci. Rep. 2015, 5, 17558. doi:10.1038/srep17558

39. Conley, H. J.; Wang, B.; Ziegler, J. I.; Haglund, R. F., Jr.; Pantelides, S. T.; Bolotin, K. I. Nano Lett. 2013, 13, 3626-3630. doi:10.1021/nl4014748

40. Feng, J.; Qian, X.; Huang, C.-W.; Li, J. Nat. Photonics 2012, 6 , 866-872. doi:10.1038/nphoton.2012.285

41. Ji, Q.; Zhang, Y.; Gao, T.; Zhang, Y.; Ma, D.; Liu, M.; Chen, Y.; Qiao, X.; Tan, P.-H.; Kan, M.; Feng, J.; Sun, Q.; Liu, Z. Nano Lett. 2013, 13, 3870-3877. doi:10.1021/nl401938t

42. Wang, Y. G.; Zhang, Q. L.; Wang, T. H.; Han, W.; Zhou, S. X. J. Phys. D: Appl. Phys. 2011, 44, 125301. doi:10.1088/0022-3727/44/12/125301

43. Zhou, L.; Zhuo, Z.; Kou, L.; Du, A.; Tretiak, S. Nano Lett. 2017, 17, 4466-4472. doi:10.1021/acs.nanolett.7b01704

44. Tran, V.; Soklaski, R.; Liang, Y.; Yang, L. Phys. Rev. B: Condens. Matter Mater. Phys. 2014, 89, 235319. doi:10.1103/PhysRevB.89.235319

45. Choi, J.-H.; Cui, P.; Lan, H.; Zhang, Z. Phys. Rev. Lett. 2015, 115 , 066403. doi:10.1103/PhysRevLett.115.066403

46. Omelchenko, S. T.; Tolstova, Y.; Atwater, H. A.; Lewis, N. S. Excitonic effects in photovoltaic materials with large exciton binding energies. In 2016 IEEE 43rd Photovoltaic Specialists Conference (PVSC), Portland, OR, U.S.A., June 5-10, 2016; IEEE Publishing: Piscataway, NJ, U.S.A., 2016. doi:10.1109/PVSC.2016.7750347 


\section{License and Terms}

This is an Open Access article under the terms of the Creative Commons Attribution License

(http://creativecommons.org/licenses/by/4.0), which permits unrestricted use, distribution, and reproduction in any medium, provided the original work is properly cited.

The license is subject to the Beilstein Journal of Nanotechnology terms and conditions:

(https://www.beilstein-journals.org/bjnano)

The definitive version of this article is the electronic one which can be found at:

doi:10.3762/bjnano.9.116 